\title{
Effects of Novel 3-dimensional Antibiotic-containing Electrospun Scaffolds on Dentin Discoloration
}

\author{
Margaret Louise A. Porter, DMD, MSD, ${ }^{* \dagger}$ Eliseu A. Münchow, DDS, MSD, ${ }^{*}$ \\ Maria T.P. Albuquerque, DDS, MSD, ${ }^{* \top}$ KennetbJ. Spolnik, DDS, MS, Anderson T. Hara, DDS, PbD," \\ and Marco C. Bottino, DDS, PhD*
}

\section{Ahstract}

Introduction: Although intracanal application of the triple antibiotic paste (TAP) may offer advantages (eg, disinfection), this practice has been associated with significant drawbacks, including tooth discoloration. In this study, the color change of dentin was monitored during treatment with distinct TAP pastes and novel tubularshaped 3-dimensional electrospun scaffolds containing minocycline (MINO) or doxycycline (DOX). Methods: Two TAP pastes (TAP $_{\text {MINo }}$ [MINO, metronidazole, and ciprofloxacin] and $\mathrm{TAP}_{\mathrm{DOX}}$ [DOX, metronidazole, and ciprofloxacin]), 4 scaffold-based groups containing MINO or DOX at distinct concentrations, 1 antibioticfree scaffold, and 1 untreated group (control) were investigated. Human canines were sectioned at the cementoenamel junction and tubular-shaped scaffolds or paste were placed into the root canals and sealed. Color measurements (CIEL ${ }^{*} a^{*} b^{*}$ parameters) were performed at baseline and after 1, 3, 7, 14, 21, and 28 days. Color changes were expressed as $\Delta E^{*}$ values. In addition, scanning electron microscopy and energydispersive X-ray spectroscopy were also performed on the specimens after treatment. Data were analyzed using repeated measures analysis of variance (alpha $=0.05$ ). Results: All antibiotic-containing groups led to greater discoloration than the antibiotic-free groups. A severe discoloration occurred after 1 day. At the end of the experiment, antibiotic-treated samples exhibited crusts/agglomerates over the dentin surface, which totally or partially obliterated the dentinal tubules. The presence of MINO resulted in a greater color change than DOX. Conclusions: Scaffolds containing MINO or DOX produced similar color change to dentin when compared with their respective TAP systems, although DOX-related discoloration was less pronounced. (J Endod 2016;42:106-112)

\section{Key Words}

Antibiotic, discoloration, disinfection, electrospinning, regeneration, scaffold

D ental caries and/or trauma in immature permanent teeth can lead to infection of the dental pulp, with a potential negative impact on tooth development. In the past decade, regenerative-based endodontic therapy (ie, root disinfection with the socalled triple antibiotic paste [TAP] followed by evoked bleeding) has been shown to be a viable treatment option for immature teeth with pulpal necrosis (1) because this technique allows for the replacement of damaged pulp by living tissue and tooth development (2).

An essential stage of regenerative endodontics is disinfection of the root canal via intracanal application of medicaments (eg, calcium hydroxide or the combination of antibiotics) in the form of a paste (3-5). Although intracanal treatment with TAP may offer advantages such as effective disinfection and lower probability of allergic reactions compared with systemic antibiotics, this practice has been associated with stem cell toxicity (6), development of bacterial resistance (7), and significant tooth discoloration (8-12). It has been shown that TAP produces substantial color change on dentin, especially because of minocycline (MINO) use because it can bind to the calcium ions found in dentin via chelation (13).

Several clinical reports have also confirmed that crown discoloration may appear within 24 hours of MINO application (8) and possibly after 1 hour of smear layer removal and placement of the TAP paste (12). Interestingly, a case report on a novel approach to eliminating tooth discoloration suggested that sealing the dentinal tubules of the pulp chamber with a bonding agent would be a viable alternative (14) although still not completely efficacious. Furthermore, antibiotics other than MINO (eg, doxycycline $[\mathrm{DOX}]$, cefaclor, etc.) were also shown to produce tooth discoloration when incorporated with a paste vehicle (9), thus raising an important concern regarding the use of these antibiotic-containing materials.

From a clinical perspective, the major consequence related to the discoloration produced on anterior teeth treated with TAP is esthetically driven. Additional treatments such as tooth bleaching and restorative procedures are necessary to improve visual appeal after endodontic therapy, which may increase chair time and costs for the patient. Thus, alternative therapies producing a lesser degree of tooth discoloration yet preserving the antimicrobial efficacy are paramount. In this way, polymer-based antibioticcontaining scaffolds recently have been shown to be a promising method for attaining effective disinfection $(4,15-17)$. Notably, the antibiotic concentration incorporated

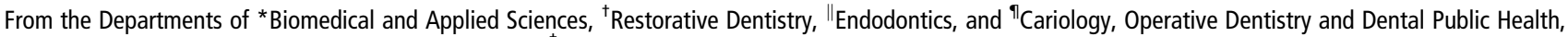
Indiana University School of Dentistry, Indianapolis, Indiana; ${ }^{\ddagger}$ Department of Operative Dentistry, Federal University of Pelotas, School of Dentistry, Pelotas, Rio Grande do Sul, Brazil; and ${ }^{\S}$ Universidade Estadual Paulista, São José dos Campos Dental School, São José dos Campos, São Paulo, Brazil.

Address requests for reprints to Dr Marco C. Bottino, Department of Biomedical and Applied Sciences, Indiana University School of Dentistry, 1121 West Michigan Street (DS270B), Indianapolis, IN 46202. E-mail address: mbottino@iu.edu

0099-2399/\$ - see front matter

Copyright (๐ 2016 American Association of Endodontists.

http://dx.doi.org/10.1016/j.joen.2015.09.013 
into these scaffolds is considerably lower when compared with the concentration used in conventional TAP $(4,18)$. Therefore, the following hypotheses were tested:

1. The use of novel tubular-shaped 3-dimensional (3D) antibioticcontaining electrospun scaffolds would produce less or no discoloration on dentin when compared with the clinically advocated TAP paste.

2. The presence of MINO would produce greater color change when compared with an alternative antibiotic (ie, DOX).

\section{Study Design}

\section{Materials and Methods}

This study evaluated the effects of antibiotic-based delivery systems (ie, TAP pastes containing different combinations of antibiotics or tubular-shaped 3D antibiotic-containing scaffolds) on the color change of human dentin. Eight groups were investigated according to the ma-

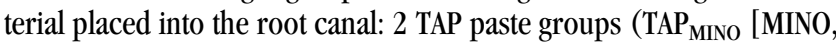
metronidazole (MET), and ciprofloxacin (CIP)] and TAP Dox [DOX, MET, and CIP]); 4 groups of antibiotic-containing scaffolds (MINO or DOX); 1 group of an antibiotic-free scaffold (Scaffold) to serve as the control for the novel scaffolds; and 1 group to act as the control for the TAP paste groups (saline, $0.9 \%$ sodium chloride irrigation; Baxter Healthcare Corporation, Deerfield, IL). Forty specimens were tested in total, which were evaluated with a chroma meter at different time intervals up to 28 days and have color change $\left(\Delta E^{*}\right)$ as the study's outcome.

\section{Synthesis of Tubular-shaped 3D Antibiotic-containing Electrospun Scaffolds}

Polydioxanone polymer solutions (PDS II; Ethicon, Somerville, $\mathrm{NJ}$ ) were prepared by dissolving PDS in 1,1,1,3,3,3-hexafluoro-2propanol at a concentration of $10 \mathrm{wt} \%$. Each solution was then incorporated with MINO (Sigma-Aldrich, St Louis, MO) or DOX (Sigma-Aldrich) at 25 or $50 \mathrm{wt} \%$ (respective to the total polymer weight). Antibiotic-free PDS solution was also prepared to serve as the control. Each solution was spun (ie, flow rate of $2 \mathrm{~mL} / \mathrm{h}, 18-\mathrm{cm}$ distance, and electrical voltage between 15 and $19 \mathrm{kV}$ ) into nanofibers using a conventional electrospinning system and collected on a custommade grounded Teflon-coated stainless steel mandrel (KECO Coatings, Inc, Indianapolis, IN) $(2.0 \pm 0.02 \mathrm{~mm})$ connected to a high-speed stirrer (BDC6015; Caframo, Wiarton, ON) at 120 revolutions per minute. After processing, the scaffolds (Fig. $1 A$ and $B$ ) were cut into tubularshaped specimens $(6.0-\mathrm{mm}$ height $\pm 0.1 \mathrm{~mm})$ and dried under a vacuum to remove any remaining solvent $(15,16)$.

\section{Tooth Sample Preparation and Initial Color Measurements [Baseline]}

Forty human canines were sectioned $3 \mathrm{~mm}$ above and $5 \mathrm{~mm}$ below the cementoenamel junction to obtain 8.0 -mm-thick $( \pm 0.1 \mathrm{~mm})$ enamel/dentin samples (8). The smear layer was removed by sample immersion in $2.5 \%$ sodium hypochlorite ( $\mathrm{NaOCl}$ ) solution for 3 minutes followed by 3 baths of distilled water (DI) and then immersion in 17\% EDTA (Inter-Med, Inc, Racine, WI) for 3 minutes. The apical foramen of all tooth samples was closed using a bonding system (Adper Scotchbond Multi-Purpose; 3M ESPE, St Paul, MN) and resin composite (Z100, 3M ESPE). After that, each specimen was then stored in DI until use. The color of all specimens was evaluated under a chroma meter (Minolta CR-241; Konica Minolta Sensing Americas, Inc, Ramsey, NJ) considering the aperture size of $0.3 \mathrm{~mm}$ (smaller measurable circle, Fig. $1 E$ ); the 3 most anterior areas of the tooth were measured using the CIE $L^{*} a^{*} b^{*}$ color system, and the 3 measurements were then averaged. As shown in Figure $1 F-H$, the wall of the root canal of each sample was used as the initial parameter to determine the location of the bigger measurable circle $(1.8 \mathrm{~mm})$, thus facilitating the measurement of the same area at each time point investigated.

\section{Application of Testing Materials and Final Color Measurements}

The 3D tubular-shaped electrospun scaffolds were weighed $(\mathrm{S}+\mathrm{DOX} 25 \%=9.48 \pm 2.36 \mathrm{mg}[\mathrm{DOX}=2.37 \pm 0.59 \mathrm{mg}]$, $\mathrm{S}+$ DOX $50 \%=7.54 \pm 0.91 \mathrm{mg}[\mathrm{DOX}=3.77 \pm 0.46 \mathrm{mg}]$, $\mathrm{S}+\mathrm{MINO25 \%}=6.60 \pm 0.86 \mathrm{mg}$ [MINO $=1.65 \pm 0.22 \mathrm{mg}$ ], and $\mathrm{S}+\mathrm{MINO} 50 \%=9.62 \pm 0.52 \mathrm{mg}[\mathrm{MINO}=4.76 \pm 0.26 \mathrm{mg}])$ and directly placed into the root canal, sized to match the diameter, and perfectly (no gaps) adapted to the canal walls (Fig. 1C). Standardized preparation of the root canal was performed by instrumentation up to Kfile \#40 and Gates Glidden \#6 and enlargement with a round bur $(\phi=2.7 \mathrm{~mm})$. Meanwhile, the antibiotic pastes were prepared by mixing the respective antibiotics MET (Sigma-Aldrich), CIP, and MINO $\left(\mathrm{TAP}_{\mathrm{MINO}}\right)$ or DOX (TAP $\left.{ }_{\mathrm{DOX}}\right)$ with saline at the clinically used concentration $(1 \mathrm{~g} / \mathrm{mL})$ and then placed into the root canal with a sterile Lentulo spiral filler. One group of specimens was filled with saline (control). The root canals were then temporary sealed (Cavit, 3M ESPE). All samples were stored inside 24 -well plates at $37^{\circ} \mathrm{C}$. A wet environment was created to mimic the root canal condition. Briefly, nonsticky wax was placed on the bottom surface of the plate to stabilize the tooth sample. All samples were stored for 28 days and kept in direct contact with the testing materials. Color measurements were taken on days $1,3,7,14,21$, and 28 (Fig. $1 D$ and $E$ ).

\section{Color Change Analysis}

The color change $\left(\Delta E^{*}\right)$ of each sample (ie, an average of the 3 preselected tested areas, Fig. $1 F-H)$ and at each time interval was assessed using the following formula:

$$
\Delta E^{*}=\sqrt{\left(\Delta L^{*}\right)^{2}+\left(\Delta a^{*}\right)^{2}+\left(\Delta b^{*}\right)^{2}}
$$

where $\Delta L^{*}, \Delta a^{*}$, and $\Delta b^{*}$ corresponded, respectively, to the difference between the test days and baseline $L^{*}, a^{*}$, and $b^{*}$ values.

\section{Scanning Electron Microscopy and Energy-dispersive X-ray Spectroscopy}

After 28 days, 2 samples from each group were randomly allocated for scanning electron microscopic analysis. The teeth were mesiodistally cleaved with a size 5 LeCron instrument (Hu-Friedy Mfg Co, LLC, Chicago, IL) in 2 halves. Although 1 of the halves was cleaned with DI water to evaluate the dentin surface, the other half was kept untouched to analyze the overall aspects of the material placed inside the root canal (pastes or scaffolds). The specimens were sputter coated with Au-Pd before imaging (FE-SEM, Model JSM-6701F; JEOL Ltd, Tokyo, Japan). Energy-dispersive X-ray spectroscopic (EDS) analysis was done to study the chemical composition of the dentin surface and materials immediately after their removal from the canal.

\section{Statistical Analysis}

The quantitative color change $\left(\Delta E^{*}\right)$ data were statistically analyzed (SigmaPlot version 12; Systat Software Inc, San Jose, CA, USA) using repeated measures analysis of variance. Time was repeated within each sample, and group was a between-sample factor. The Sidak 


\section{Regenerative Endodontics}
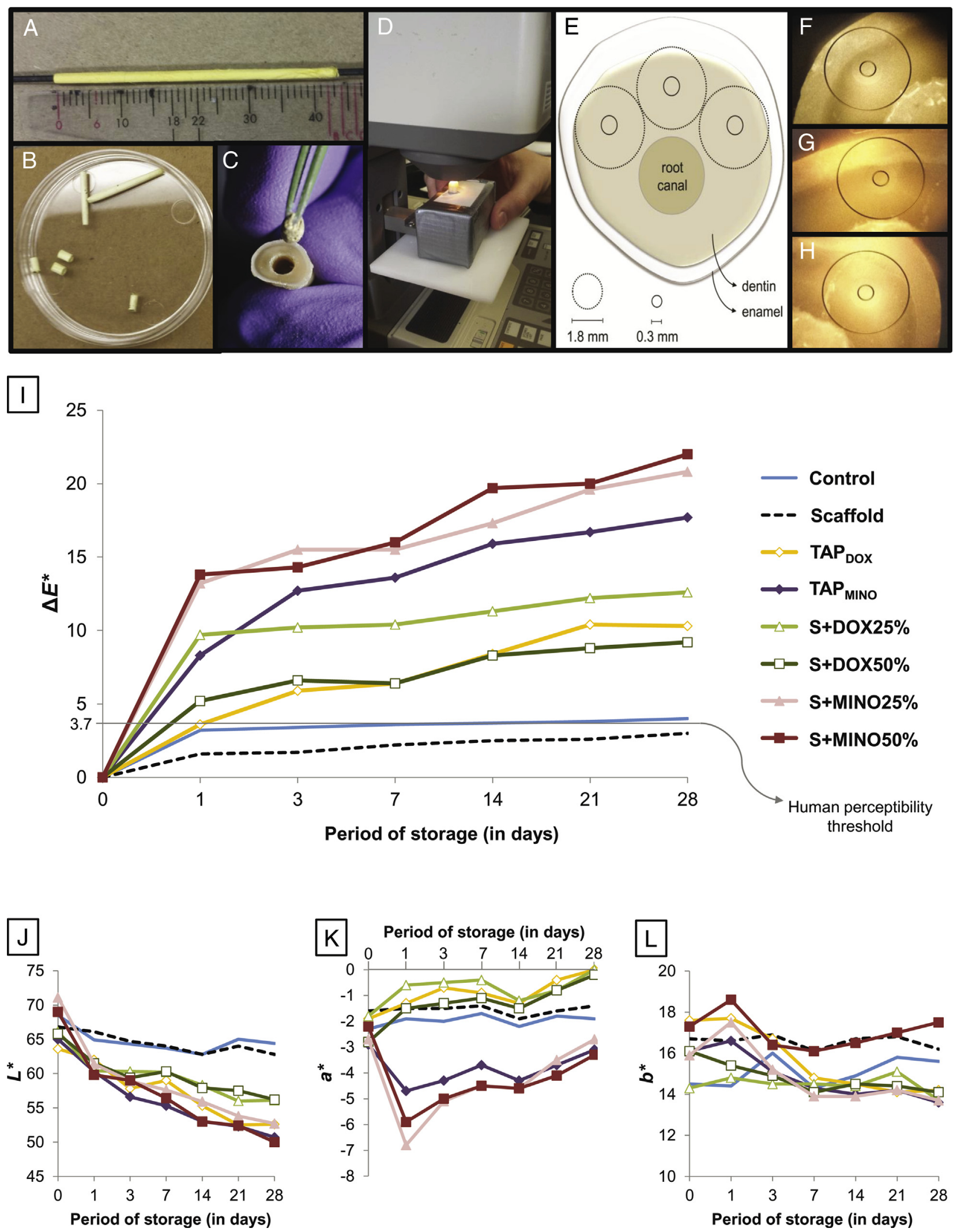

Figure 1. $(A)$ Image showing the Teflon-coated stainless steel rod used to prepare the 3D tubular-shaped electrospun scaffolds. $(B$ and $C$ ) Images showing the appearance of the 6-mm-long scaffolds and placement into the root canal. $(D)$ The chroma meter used in the present study. $(E)$ A schematic diagram showing the 3 preselected anterior areas of each specimen used to measure the color of dentin. $(F-H)$ An actual experimental view of the preselected areas at $0.3 \mathrm{~mm}$. $(I) \mathrm{Graph}$ showing the progression of $\Delta E^{*}$ values with increased days of storage. $(J-L)$ Graphs showing changes in the $L^{*}, a^{*}$, and $b^{*}$ color parameters of each group at each time point tested. 
method was used to control the overall significance level of the pairwise group comparisons at $5 \%$ for each time point and location.

\section{Results}

The color change results obtained in the study are shown in Figures $1 I$ and $2 A$. According to the statistical analysis, both factors "material" and "storage period" were significant $(P \leq .001)$ although their interaction was not $(P=.462)$. Overall, groups containing MINO exhibited considerably higher color change than groups containing DOX; additionally, all antibiotic-based groups resulted in greater discoloration compared with the antibiotic-free groups $(P \leq .001)$ (Fig. $2 A)$. A severe discoloration (beyond the human perceptibility threshold, ie, $\Delta E^{*}=3.7$ ) (9) occurred after the first day of placement of all antibiotic-containing materials (Fig. 1I). Although the discoloration effect clearly increased with greater periods of storage, specimens treated with S + DOX25\%, S + MINO25\%, and $\mathrm{S}+$ MIN050\% showed similar $\Delta E^{*}$ values after 28 days (Fig. $2 A$ ) when compared with 1 day of storage $(P \geq .098)$. Related to the individual color parameters tested (Fig. $1 J-L$ ), all groups became darker (reduction in the $L^{*}$ axis); however, it was more intensive for groups treated with antibiotics. Specimens treated with MINO-based materials exhibited reduction of the $a^{*}$ axis (ie, they became greenish), especially after day 1, whereas specimens treated with DOX-based materials slightly increased the $a^{*}$ axis (Fig. $1 K$ ). The $b^{*}$ axis was not pointedly affected by the tested materials (Fig. $1 L$ ).

\begin{tabular}{|c|c|c|c|c|c|c|}
\hline \multirow{2}{*}{ A } & \multicolumn{6}{|c|}{ Period of storage (in days) } \\
\hline & 1 & 3 & 7 & 14 & 21 & 28 \\
\hline Control & ${ }^{D} 3.2( \pm 0.1)^{\mathrm{a}}$ & ${ }^{c} 3.4( \pm 0.3)^{a}$ & ${ }^{D} 3.6( \pm 0.2)^{a}$ & ${ }^{\circ} 3.7( \pm 0.2)^{\mathrm{a}}$ & $E_{3.8}( \pm 0.5)^{a}$ & ${ }^{D} 4.0( \pm 0.4)^{\mathrm{a}}$ \\
\hline Scaffold (S) & $\mathrm{E}_{1.6}( \pm 0.5)^{\mathrm{b}}$ & ${ }^{D} 1.7( \pm 0.5)^{b}$ & $\mathrm{E}_{2.2}( \pm 0.6)^{\mathrm{ab}}$ & ${ }^{\mathrm{D}} 2.5( \pm 0.5)^{\mathrm{ab}}$ & $\mathrm{E} 2.6( \pm 0.7)^{\mathrm{ab}}$ & D3.0 $( \pm 0.7)^{\mathrm{a}}$ \\
\hline TAP $_{\text {Dox }}$ & D3.6 $( \pm 1.1)^{\mathrm{c}}$ & ${ }^{8} 5.9( \pm 1.7)^{\mathrm{ab}}$ & ${ }^{C D} 6.4( \pm 1.9)^{\mathrm{bc}}$ & ${ }^{c} 8.4( \pm 2.4)^{\mathrm{ab}}$ & $C D 10.4( \pm 1.3)^{a}$ & 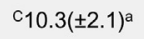 \\
\hline TAP $_{\text {MINO }}$ & ${ }^{\mathrm{BC}} 8.3( \pm 2.6)^{\mathrm{b}}$ & ${ }^{A} 12.7( \pm 3.8)^{\mathrm{ab}}$ & ${ }^{A} 13.6( \pm 3.6)^{\mathrm{ab}}$ & АB $15.9( \pm 4.2)^{\mathrm{a}}$ & $\mathrm{ABC}_{16} 6.7( \pm 5.0)^{\mathrm{a}}$ & $\mathrm{AB} 17.7( \pm 5.0)^{\mathrm{a}}$ \\
\hline S+DOX25\% & ${ }^{A B} 9.7( \pm 2.9)^{\mathrm{a}}$ & $\mathrm{AB} 10.2( \pm 3.0)^{\mathrm{a}}$ & $\mathrm{AB}_{10.4}( \pm 3.1)^{\mathrm{a}}$ & $\mathrm{BC}_{11} .3( \pm 3.3)^{\mathrm{a}}$ & $\mathrm{BCD} 12.2( \pm 3.3)^{\mathrm{a}}$ & $\mathrm{BC}_{12.6}( \pm 3.6)^{\mathrm{a}}$ \\
\hline S+DOX50\% & $\operatorname{CD} 5.2( \pm 1.5)^{b}$ & ${ }^{\mathrm{B}} 6.6( \pm 1.9)^{\mathrm{ab}}$ & ${ }^{\mathrm{BC}} 6.4( \pm 1.4)^{\mathrm{ab}}$ & ${ }^{c} 8.3( \pm 2.4)^{\mathrm{ab}}$ & $\mathrm{D} 8.8( \pm 2.6)^{\mathrm{ab}}$ & ${ }^{c} 9.2( \pm 2.7)^{\mathrm{a}}$ \\
\hline S+MINO25\% & ${ }^{A B} 13.2( \pm 3.6)^{a}$ & ${ }^{A} 15.5( \pm 2.5)^{\mathrm{a}}$ & $\mathrm{A} 15.5( \pm 2.9)^{\mathrm{a}}$ & $\mathrm{AB} 17.3( \pm 3.1)^{\mathrm{a}}$ & $A B 19.6( \pm 3.1)^{\mathrm{a}}$ & ${ }^{A} 20.8( \pm 4.0)^{\mathrm{a}}$ \\
\hline S+MINO50\% & $\mathrm{A} 13.8( \pm 3.9)^{\mathrm{a}}$ & ${ }^{A} 14.3( \pm 3.3)^{\mathrm{a}}$ & $\mathrm{A}_{16.0}( \pm 3.5)^{\mathrm{a}}$ & $\mathrm{A}_{19.7}( \pm 5.0)^{\mathrm{a}}$ & ${ }^{A} 20.0( \pm 4.6)^{\mathrm{a}}$ & ${ }^{A} 22.0( \pm 4.7)^{\mathrm{a}}$ \\
\hline
\end{tabular}

Distinct superscript uppercase letters before means (in a same column) and lowercase letters after \pm SD (in a same row) indicate statistically significant differences among groups and periods of storage, respectively $(p<0.05)$.

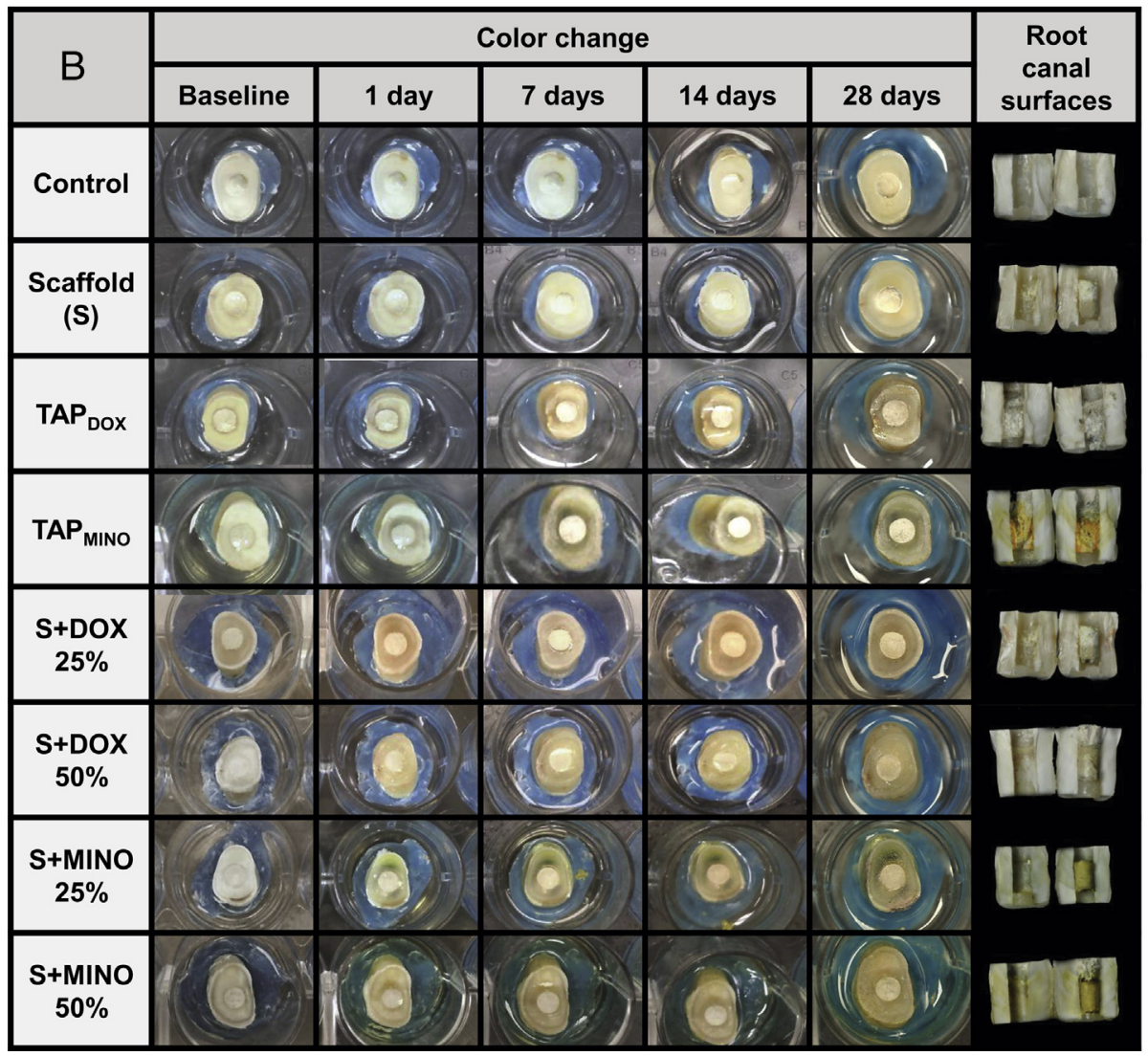

Figure 2. (A) Table showing the color change $\left(\Delta E^{*}\right)$ means ( \pm standard deviation) for all groups investigated including control (saline), scaffold ( $\left.\mathrm{S}\right)$ based on polydioxanone/PDS, triple antibiotic paste/TAP prepared with doxycycline/DOX or minocycline/MINO, and scaffolds containing 25 or $50 \mathrm{wt} \%$ of DOX or MINO at different periods of storage. $(B)$ Representative macrophotographs showing the color stability/change of specimens after $1,7,14$, and 28 days of storage. 
Representative macrophotographs of tooth samples treated with the distinct antibiotic(s) delivery methods at each time point investigated, except after 3 and 21 days of storage, are presented in Figure $2 B$. Although untreated specimens (control) and those treated with the antibiotic-free scaffold (S) exhibited no dentin discoloration, specimens treated with antibiotic-containing materials suffered a visually circumferential discoloration. The images also show that samples treated with MINO-based materials became greenish, whereas those treated with DOX became brownish. With regard to the root canal surfaces at the end of the experiment, surfaces treated with TAP ${ }_{\text {MINO }}$ showed the most dentin discoloration.

Figure 3 shows the SEM micrographs and EDS spectra of the dentin surface after direct contact with the tested materials for 28 days as well as the surface of the materials used. Untreated specimens presented completely open dentinal tubules (no obliteration) and showed intense detection of calcium and phosphorus. In contrast, specimens treated with scaffolds and $\mathrm{TAP}_{\mathrm{DOx}}$ showed partial obliteration of the tubules and a vast amount of organic residues (carbon-based compounds). Specimens treated with $\mathrm{TAP}_{\text {MINO }}$ exhibited nearly complete obliteration of the tubules and the presence of elements, such as zinc, calcium, phosphorus, and tellurium. Other chemical elements were also observed on the S + MINO50\%-treated specimens. Thick crusts could be seen attached to the dentin surface of all specimens treated with antibiotics. Regarding the material's surface, all scaffolds appeared to be moderately intact after 28 days of storage. Within the TAP ${ }_{\text {Dox }}$ paste, ferule-shaped powder composed of carbon and oxygen was observed, whereas rodlike powder composed of carbon, oxygen, and chlorine was noted within the TAP $\mathrm{TINO}_{\text {paste. }}$

\section{Discussion}

The first hypothesis of this study was to determine whether the use of novel tubular-shaped 3D antibiotic-containing scaffolds would produce less or no discoloration on dentin when compared with the traditionally used TAP paste (ie, TAP prepared with MINO). Based on the reported findings, this hypothesis was rejected. Surprisingly, both antibiotic vehicles (ie, scaffold or paste) led to similar $\Delta E^{*}$ values at the end of the experiment (Fig. 1A). Considering the scaffold vehicle carried a much smaller amount (fewer milligrams per scaffold) of antibiotics than the TAP vehicle $(1 \mathrm{~g} / \mathrm{mL})$, the former was supposed to provoke less discoloration than the latter. Additionally, even the more concentrated scaffold group (S + MINO50\%) did not cause greater discoloration compared with the less concentrated group ( $\mathrm{S}+\mathrm{MINO25 \% )}$. Thus, it can be suggested that antibiotic concentration does not seem to be the major factor related to tooth discoloration, but, more importantly, the nature/composition of the antibiotic(s) used emerges as the leading determinant. This inference can also be reinforced by the results obtained for the DOX-containing groups; a similar color change was obtained regardless of the vehicle and concentration factors. The replacement of MINO with DOX resulted in a significant reduction in the total color change although, from an esthetics standpoint, still clinically undesirable because it would require additional procedures (eg, bleaching) in order to treat the resultant discoloration. Even so, and despite this unsatisfactory finding, it is worth mentioning that DOX has a lower discoloration potential compared with MINO; thus, the second study hypothesis was accepted.

A recent study by Akcay et al (9) found similar results to the ones reported herein (ie, the presence of MINO was associated with greater discoloration when compared with DOX). This might account for the ability of MINO to bind to the calcium ions of dentin, causing a chelation reaction that stains the substrate (13); alternatively, DOX has not yet been shown to have the same ability. Nonetheless, a chemical reaction between a root canal irrigant containing DOX (BioPure MTAD; Dentsply, Tulsa, OK) and distinct concentrations of $\mathrm{NaOCl}$ leads to redpurple staining of human teeth (19). It can be suggested that the DOX used in this study somehow interacted with residual NaOCl molecules left in the dentin, thus leading to staining (Fig. 2B). Considering that both MINO and DOX are derivatives of the same substance (ie, tetracycline), there is also the possibility that DOX has some chelation potential, similar to MINO. This should be the focus of future research using appropriated methodologies.

Several studies have suggested modifying the formulation of the TAP paste by replacing MINO with alternative antibiotics (9, 20-22) or reducing treatment duration from 21-28 days to 24-48 hours (8). Nonetheless, both strategies do not seem to be completely effective in avoiding severe dentin discoloration as shown by the collected data. Moreover, using a double antibiotic paste constituted of MET and CIP has been considered. Although this strategy would effectively prevent dentin discoloration (9), questions remain regarding its overall disinfection potential and clinical prognosis because only a few clinical cases have used this antibiotic combination. It is worth mentioning that calcium hydroxide has been used as an intracanal medicament for regenerative endodontics $(23,24)$, and according to a recent study (10), it did not produce significant color change on dentin-enamel samples; consequently, calcium hydroxide may be considered for disinfection purposes because it does not jeopardize the color appearance.

The antimicrobial potential of novel TAP-mimic scaffolds has recently been shown against an Actinomyces naeslundii (4) biofilm, a very prevalent microorganism found in necrotic permanent immature teeth (25). The formation of calcium-enriched agglomerates over the dentin surface was also seen in a former study causing staining of the substrate, a phenomenon that was attributed to the presence of MINO (4). It can be speculated that the aforementioned agglomerates would affect not only the tooth color but also the structural and morphologic characteristics of dentin. These characteristics could have implications in the regenerative outcome. In this study, all specimens treated with antibiotic(s) exhibited the presence of crusts/agglomerates partially covering the dentin surface (Fig. 3). These agglomerates showed a heterogeneous composition (EDS analyses) although calcium elements could also be detected, corroborating the results of a previous study (4). Once again, it can be noticed that even the less concentrated scaffold groups resulted in the formation of these crusts, denoting that the contact of the antibiotic(s) with the substrate, regardless of the concentration or vehicle used, is sufficient to form these mineralized agglomerates. Considering that MINO and DOX have a very small structure (please note the SEM micrographs showing the shape and size of the antibiotic powders), it can be speculated that antibiotics can interact easily with the dentin components, forming complex agglomerates that may serve as an antibiotic(s) reservoir. Although one might view this reservoir ability as an advantage as it pertains to the antibacterial potential of the treatment, the application of antibiotics into the root canal is known to negatively affect the survival of stem cells (6), and it changes the morphologic characteristics of radicular dentin (26), possibly limiting their use. It is shown in the SEM micrographs that specimens treated with antibiotics revealed the dentinal tubules as obliterated by these crusts, considerably reducing dentin permeability. Conversely, the specimens that did not receive any antibiotic treatment exhibited no tubule obliteration (control) or only slight obliteration (scaffold) (Fig. 3).

The use of antibiotic-containing scaffolds during regenerative endodontic therapy is quite new in dentistry and should be investigated further in order to clarify the effects of these biomaterials on dentin properties. Although the experimental scaffolds containing MINO or DOX did not prevent dentin discoloration, this strategy carries clinical 
Regenerative Endodontics

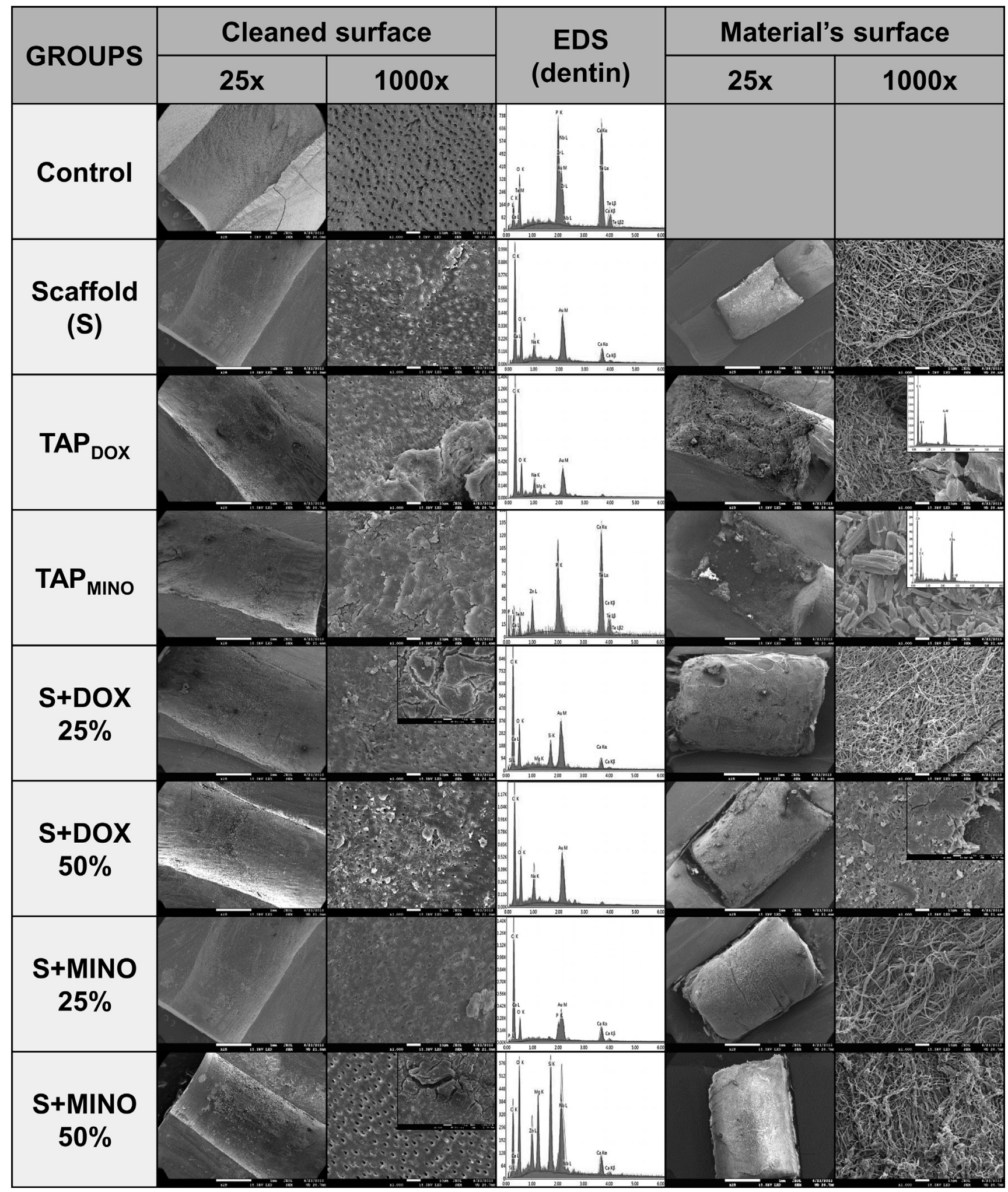

Figure 3. SEM micrographs and EDS spectra showing the morphology and superficial chemical composition of dentin (cleaned surface) and the tested materials (material's surface) after 28 days of experiment. Untreated specimens (control) did not show dentinal tubule obliteration; calcium and phosphorus were intensively detected. Specimens treated with scaffolds and $\mathrm{TAP}_{\mathrm{Dox}}$ showed partial dentinal tubule obliteration and a vast amount of organic residues (carbon-based compounds). Specimens treated with $\mathrm{TAP}_{\mathrm{MINO}}$ exhibited nearly complete dentinal tubules obliteration and the presence of elements, such as zinc, calcium, phosphorus, and tellurium. Thick crusts could be seen attached to the dentin surface of all specimens treated with antibiotics. Regarding the material's surface, all scaffolds appeared to be moderately intact after 28 days of storage. 
potential for the treatment of necrotic teeth. The replacement of MINO by other antibiotics, such as amoxycillin and clavulanic acid (27), is under investigation as a potential discoloration-free strategy. The method used in this study to measure color change was based on the CIE $L^{*} a^{*} b^{*}$ color system, which is a device-independent system. Although it has been considered the universal method to assess color changes that are easily perceptible to the human eye, one should bear in mind that the use of an alternative system such as the RGB color space might have produced different results. Nonetheless, the combination of the quantitative $\left(\Delta E^{*}\right)$ and qualitative (macrophotographs) data allowed for a satisfactory collection of important information regarding the dentin discoloration potential of antibiotics used for regenerative endodontics.

Taken together, scaffolds containing MINO or DOX produced similar color changes to dentin when compared with their respective TAP paste systems. Materials containing DOX suffered less discoloration than materials prepared with MINO. Both drugs were associated with the formation of crusts/agglomerates over the radicular dentin surface.

\section{Acknowledgments}

Margaret Louise A. Porter and Eliseu A. Münchow contributed equally to this study. statistics.

The authors are grateful to Mr George J. Eckert for bis belp in

Marco C. Bottino acknowledges funding from an International Development Funds (IDF) Grant from Indiana University Purdue University (IUPUI/OVCR) and start-up funds from Indiana University School of Dentistry (IUSD).

The authors deny any conflicts of interest related to this study.

\section{References}

1. Murray PE, Garcia-Godoy F, Hargreaves KM. Regenerative endodontics: a review of current status and a call for action. J Endod 2007;33:377-90.

2. Bose R, Nummikoski P, Hargreaves K. A retrospective evaluation of radiographic outcomes in immature teeth with necrotic root canal systems treated with regenerative endodontic procedures. J Endod 2009;35:1343-9.

3. Raju SM, Yadav SS, Kumar MS. Revascularization of immature mandibular premolar with pulpal necrosis - a case report. J Clin Diagn Res 2014;8:ZD29-31.

4. Albuquerque MT, Ryan SJ, Munchow EA, et al. Antimicrobial effects of novel triple antibiotic paste-mimic scaffolds on Actinomyces naeslundii biofilm. J Endod 2015: 41:1337-43.

5. Banchs F, Trope M. Revascularization of immature permanent teeth with apical periodontitis: new treatment protocol? J Endod 2004;30:196-200.

6. Ruparel NB, Teixeira FB, Ferraz CC, et al. Direct effect of intracanal medicaments on survival of stem cells of the apical papilla. J Endod 2012;38:1372-5.
7. Bottino MC, Arthur RA, Waeiss RA, et al. Biodegradable nanofibrous drug delivery systems: effects of metronidazole and ciprofloxacin on periodontopathogens and commensal oral bacteria. Clin Oral Investig 2014;18:2151-8.

8. Kim JH, Kim Y, Shin SJ, et al. Tooth discoloration of immature permanent incisor associated with triple antibiotic therapy: a case report. J Endod 2010;36:1086-91.

9. Akcay M, Arslan H, Yasa B, et al. Spectrophotometric analysis of crown discoloration induced by various antibiotic pastes used in revascularization. J Endod 2014;40: 845-8.

10. Lenherr $\mathrm{P}$, Allgayer $\mathrm{N}$, Weiger $\mathrm{R}$, et al. Tooth discoloration induced by endodontic materials: a laboratory study. Int Endod J 2012;45:942-9.

11. Petrino JA, Boda KK, Shambarger S, et al. Challenges in regenerative endodontics: a case series. J Endod 2010;36:536-41.

12. Ahmed HM, Abbott PV. Discolouration potential of endodontic procedures and materials: a review. Int Endod J 2012;45:883-97.

13. Tanase $\mathrm{S}$, Tsuchiya $\mathrm{H}$, Yao J, et al. Reversed-phase ion-pair chromatographic analysis of tetracycline antibiotics. Application to discolored teeth. J Chromatogr B Biomed Sci Appl 1998;706:279-85.

14. Reynolds K, Johnson JD, Cohenca N. Pulp revascularization of necrotic bilateral bicuspids using a modified novel technique to eliminate potential coronal discolouration: a case report. Int Endod J 2009;42:84-92.

15. Bottino MC, Kamocki K, Yassen GH, et al. Bioactive nanofibrous scaffolds for regenerative endodontics. J Dent Res 2013;92:963-9.

16. Bottino MC, Yassen GH, Platt JA, et al. A novel three-dimensional scaffold for regenerative endodontics: materials and biological characterizations. J Tissue Eng Regen Med 2015;9:E116-23.

17. Kim HW, Knowles JC, Kim HE. Development of hydroxyapatite bone scaffold for controlled drug release via poly(epsilon-caprolactone) and hydroxyapatite hybrid coatings. J Biomed Mater Res B Appl Biomater 2004;70:240-9.

18. Kamocki K, Nor JE, Bottino MC. Dental pulp stem cell responses to novel antibioticcontaining scaffolds for regenerative endodontics. Int Endod J 2015;48:1147-56.

19. Tay FR, Mazzoni A, Pashley DH, et al. Potential iatrogenic tetracycline staining of endodontically treated teeth via NaOCl/MTAD irrigation: a preliminary report. J Endod 2006;32:354-8.

20. Sato I, Ando-Kurihara N, Kota K, et al. Sterilization of infected root-canal dentine by topical application of a mixture of ciprofloxacin, metronidazole and minocycline in situ. Int Endod J 1996;29:118-24.

21. Tawfik H, Abu-Seida AM, Hashem AA, et al. Regenerative potential following revascularization of immature permanent teeth with necrotic pulps. Int Endod J 2013;46: 910-22.

22. Trope M. Treatment of the immature tooth with a non-vital pulp and apical periodontitis. Dent Clin North Am 2010;54:313-24.

23. Cehreli ZC, Isbitiren B, Sara S, Erbas G. Regenerative endodontic treatment (revascularization) of immature necrotic molars medicated with calcium hydroxide: a case series. J Endod 2011;37:1327-30.

24. Lee BN, Moon JW, Chang HS, et al. A review of the regenerative endodontic treatment procedure. Restor Dent Endod 2015;40:179-87.

25. Nagata JY, Soares AJ, Souza-Filho FJ, et al. Microbial evaluation of traumatized teeth treated with triple antibiotic paste or calcium hydroxide with $2 \%$ chlorhexidine gel in pulp revascularization. J Endod 2014;40:778-83.

26. Yassen GH, Chu TM, Eckert G, et al. Effect of medicaments used in endodontic regeneration technique on the chemical structure of human immature radicular dentin: an in vitro study. J Endod 2013;39:269-73.

27. Bezgin T, Yilmaz AD, Celik BN, et al. Efficacy of platelet-rich plasma as a scaffold in regenerative endodontic treatment. J Endod 2015;41:36-44. 